Bryn Mawr College

Scholarship, Research, and Creative Work at Bryn Mawr College

Psychology Faculty Research and Scholarship

Psychology

2013

\title{
Peer Influences on Adolescent Risk Behavior
}

Dustin Albert

Bryn Mawr College, walbert@brynmawr.edu

Jason Chein

Laurence Steinberg

Let us know how access to this document benefits you.

Follow this and additional works at: http://repository.brynmawr.edu/psych_pubs

Part of the Psychology Commons

\section{Custom Citation}

Albert, D., Chein, J., \& Steinberg, L. 2013. Peer influences on adolescent decision making. Current Directions in Psychological Science, 22.2, 114-120.

This paper is posted at Scholarship, Research, and Creative Work at Bryn Mawr College. http://repository.brynmawr.edu/psych_pubs/57

For more information, please contact repository@brynmawr.edu. 
Peer Influences on Adolescent Risk Behavior

Dustin Albert and Laurence Steinberg

Temple University 


\begin{abstract}
Moving beyond studies of age differences in "cool" cognitive processes related to risk perception and reasoning, new approaches to understanding adolescent risk behavior highlight the influence of "hot" social and emotional factors on adolescents' decisions. Building on evidence from developmental neuroscience, we present a theory that highlights an adolescent gap in the developmental timing of neurobehavioral systems underpinning incentive processing and cognitive control. Whereas changes in brain regions involved in incentive processing result in heightened sensitivity to social and emotional rewards in early adolescence, cognitive control systems do not reach full maturity until late adolescence or early adulthood. Within this framework, middle adolescence represents a window of heightened vulnerability to peer influences toward risk-taking behavior. At a time when adolescents spend an increasing amount of time with peers, research suggests that exposure to peer-related stimuli sensitizes the reward system to the reward value of risky behavior. As the cognitive control system gradually matures, adolescents gain the capacity to exercise self-regulation in socio-emotionally challenging situations, reflected by an increasing capacity to resist peer influence.
\end{abstract}




\section{Peer Influences on Adolescent Risk Behavior}

Evidence overwhelmingly points to adolescence as a period of heightened risk-taking in multiple domains, including experimentation with alcohol, tobacco, and drugs, unprotected sexual activity, and reckless driving (Reyna \& Farley, 2006). Although risk-taking behavior declines as youth transition into mature adult roles, the public health consequences of the adolescent spike in risky decision-making are severe. Motor-vehicle accidents are the leading cause of mortality for 15 to 20 year-olds and, despite extensive efforts to educate adolescents about the dangers of unsafe sex, rates of sexually transmitted diseases remain alarmingly high (Steinberg, 2008). Although not all individuals who initiate substance use in adolescence will progress along trajectories of abuse and addiction, most adult addicts began using substances as adolescents (Chassin, Hussong, \& Beltran, 2009). In sum, the most severe threats to adolescent health and wellbeing come not from natural causes, but rather from behavior-contingent outcomes like automobile accidents, suicide and homicide, substance abuse, and sexually transmitted diseases.

A long tradition of research in developmental psychology points to adolescents' peer groups as important contributors to trajectories of risk-taking behavior. It is well known that one of the strongest predictors of deviant behavior in adolescence is affiliation with deviant peers, and this relationship is particularly strong for adolescent substance use and abuse (Chassin et al., 2009). Crime statistics indicate that adolescents typically commit crimes, ranging from vandalism and drug use to homicide, in peer groups, whereas adults typically do so alone 
(Zimring, 1998). Furthermore, adolescents are at greater risk of being involved in an automobile accident when riding in a car with multiple adolescent passengers (Simons-Morton, Lerner, \& Springer, 2005).

Several possible explanations have been advanced to account for the association between deviant peer affiliation - or even the mere presence of peers and adolescent risk-taking behavior. First, a literal account of peer influence suggests that peer groups socialize adolescents in specific risk-taking behaviors. Research from social learning approaches like Problem Behavior Theory (Jessor \& Jessor, 1977) delineates potential pathways by which modeling and reinforcement of deviant behavior may initiate adolescents into a culture of risk-taking. Although the social learning perspective is consistent with extensive correlational evidence linking adolescent risk-taking to deviant peer affiliation, a second approach suggests that most of this association may be accounted for by selection effects or confounding variables; that is, adolescents with inclinations toward risk-taking behavior are likely to find one another, and these shared personality dispositions account for the correlations in behavior between the individual and peer group (e.g., Jaccard, Blanton, \& Dodge, 2005). A third approach accounts for the more frequent presence of peers in adolescent risk-taking situations by arguing that adolescents merely spend more time with their peers than do adults, thus increasing the probability that risk-taking tendencies are expressed in peer contexts (Brown, 2004). In the present chapter, we propose an alternative, albeit compatible, account based on experimental evidence that the mere presence of peers differentially biases adolescents toward increased risk-taking behavior (Gardner \& Steinberg, 
2005). Specifically, we propose a dual systems model of neurobehavioral development that views adolescence as a developmental window wherein the presence of peers may "prime" a reward-sensitive motivational state that frequently overwhelms the adolescent's immature capacity for inhibitory control (Steinberg, 2008).

Before presenting the rationale and evidence to support our model of peer influences on risk-taking, we first provide a brief review of traditional decisionmaking approaches to understanding increased risk behavior in adolescence. We then describe a new class of dual process theories that contrast relatively automatic ("hot") with more deliberative ("cool") modes of processing risk information, highlighting the role of affective states as inputs to the risk evaluation process. In the final section of this chapter, we review behavioral and neuroscientific evidence pointing to relatively independent trajectories of development for two core systems influencing risk-taking behavior in adolescence. The first, referred to as the socio-emotional reward system, undergoes dramatic remodeling around the time of puberty, resulting in normative increases in sensation seeking and sensitivity to socio-emotional stimuli. The second, the cognitive control system, develops in a gradual, linear pattern, and supports improvements in self-regulation observed in late adolescence and young adulthood. We present a model of adolescent risktaking that highlights the window of vulnerability created by a maturational gap between these two systems. We conclude by discussing ongoing research exploring developmental differences in the influence of peer presence on the relative engagement of the two systems in decision-making situations. 


\section{The Decision-Making Framework}

Traditional decision-making approaches, including health-belief models (e.g., Becker, 1990) and the theory of reasoned action (Ajzen \& Fishbein, 1980), emphasize that individuals behave rationally in deliberately weighing perceived risks and rewards to arrive at a decision that reflects their underlying goals (Reyna and Farley, 2006). Within this consequentialist framework, it is assumed that when individuals possess accurate information about their personal vulnerability to the consequences of risk behavior, and these risks outweigh the subjective value of the behavior, they should generate a risk-averse response (Loewenstein, Weber, Hsee, \& Welch, 2001). In short, decision-making outcomes are determined by the relative value of subjectively perceived costs and benefits, and the individual's capacity to accurately weigh these inputs against each other. It follows from this perspective that excessive risk-taking behavior in adolescence derives from a combination of the following factors: a) inaccurate perception of vulnerability to risk; b) a goal structure that overvalues the benefits of risk behavior; and c) immature cognitive processing of cost and benefit information.

Empirical work has largely failed to support these predictions. In contrast to the long-held assumption of adolescent invulnerability, adolescents perceive risks and their personal vulnerability to such risks at an equal or greater level than adults; indeed, adolescents appear to overestimate risk relative to adults (Fischhoff, 2008). Moreover, adolescents report a level of risk-aversion that is comparable to that reported by adults, which argues against an assumption of adolescent goal-structures that favor risk-taking (Reyna \& Farley, 2006). Finally, although 
risk-taking in laboratory contexts appears to decline somewhat from childhood to adulthood, children and adults use probability and outcome information in a similar fashion (Levin, Hart, \& Weller, 2007), and adolescents show logical reasoning abilities comparable to adults (Steinberg \& Cauffman, 1996). In sum, adolescents appear to possess the information and cognitive maturity to make reasoned decisions about whether to engage in risk behavior.

\section{The Role of Affect in Decision-Making}

Given the failure of traditional cognitive models to account for adolescent risk-taking, several theorists have called for increased attention to the socioemotional and contextual inputs to the decision making process (e.g., Fischhoff, 2008; Loewenstein et al., 2001; Reyna \& Farley, 2006; Steinberg, 2008). These approaches typically draw upon dual-process models to make the distinction between relatively slow, "cool," analytical processing and faster, "hot," associative, emotionally-driven processing. Whereas the rational calculus of expected value may guide decision-making in cool situations, such models have typically failed to account for decision-making in hot contexts, where social and emotional factors must be considered. Pointing out that most laboratory studies of age differences in risky decision-making have purposefully minimized socio-emotional and contextual factors, the present critique offers a simple and compelling answer to the question of why extant research has not consistently revealed differences between adolescents and adults that match the real-world evidence of heightened risky behavior in adolescence: We have been studying the wrong thing. Showing up with a group of friends to a Friday night party, many (if not most) adolescents are un- 
likely to engage in a cool deliberative process of weighing the costs and benefits of decision options and calculating the expected value of a risky choice based on known probabilities of positive and negative outcomes. Decision-making relies upon not only cognitive inputs, but also feelings: the excitement of being with friends, the thrill of crossing parental or legal boundaries, and the fear of getting caught are all plausible affective contributions to an adolescent's decision of whether to drink at the keg party. To ignore affect is to study something other than risky decision-making.

Research with adult populations has identified several pathways by which affect contributes to the decision-making process (for reviews, see Loewenstein et al., 2001; Winkielman, Knutson, Paulus, \& Trujillo, 2007). First, the anticipated emotional outcomes of behavioral alternatives contribute to cognitive assessments of their expected value (Loewenstein et al., 2001). The teenager at the keg party might imagine that joining her friends in drinking beer will lessen her social anxiety and increase her positive emotion, whereas abstaining will make her feel excluded and increase her anxiety. These anticipated emotional consequences contribute to her global evaluation of the desirability of the risky choice.

Second, direct emotional responses to qualities of the choice alternatives - that is, anticipatory emotions -- influence their evaluation, and motivate approach or avoidance behavior (Loewenstein et al., 2001). Research grounded in inferential models of the influence of emotion on cognition suggests that individuals adaptively consult their feelings as a source of information when making a judgment about a given target (e.g., "affect-as-information" (Schwarz \& Clore, 
1983)). According to the somatic marker hypothesis, such emotional guidance of behavior reflects subtle affective learning from prior experience with reinforcement and/or punishment outcomes associated with the target (Damasio, 1994). Returning to the keg party, if our teenager had a prior negative experience drinking beer, she may respond with a degree of disgust to the smell of spilled beer around the keg, and this aversive emotion might influence her behavior either indirectly (by contributing to a negative evaluation of the desirability of drinking) or directly (through heightened avoidance motivation).

A third class of affective inputs has variously been referred to as incidental emotion or background mood, and includes emotions elicited by factors not related to the decision itself (Loewenstein et al., 2001). Dating back to Zajonc's seminal affective priming studies (Zajonc, 1980), research on the interplay of emotion and cognition has demonstrated the influence of pre-existing or experimentally elicited affective states on perception, memory, judgment, and behavior (Winkielman et al., 2007). For instance, individuals surveyed on a sunny day rate their life satisfaction as higher than those contacted on a rainy day (Schwarz \& Clore, 1983), and experimental elicitation of positive or negative emotion is associated with corresponding shifts toward optimistic or pessimistic judgments about risk (Johnson \& Tversky, 1983). Importantly, recent work grounded in affective neuroscience suggests that emotions do not even need to be consciously felt to influence behavior. In a clever experiment that elicited unconscious positive or negative emotion by presenting masked happy or angry faces, participants who had viewed happy faces chose to pour and drink more of a beverage than those 
who had viewed angry faces, despite reporting no differences in subjective mood (Winkielman, Berridge, \& Wilbarger, 2005). The authors of this study argue that exposure to salient emotional stimuli, especially facial expressions, activates subcortical circuits (e.g., nucleus accumbens and/or amygdala) that project to other subcortical and cortical regions involved in incentive processing and reward valuation (see also, Winkielman et al., 2007). Thus, neural responses to emotional stimuli - whether consciously experienced or not - may modulate an individual's sensitivity to unrelated incentive stimuli, biasing the individual toward approachor avoidance-related behavior. Returning to the keg party one last time, our hypothetical teenager is likely bombarded with socio-emotional stimuli, perhaps in the form of a crowd of friends' smiling faces. This positively-valenced stimuli in turn may sensitize her reward system to respond appetitively to the incentive value of the cup of beer she is subsequently offered. In effect, her immersion in a happy crowd might sensitize her to perceive the beer as more appealing.

Despite our use of an adolescent party to illustrate these mechanisms, it is important to note that the models we have reviewed describe the influence of emotion on risk-taking behavior as observed in adult populations. It is therefore reasonable to question the models' power to account for heightened risk-taking behavior in adolescence relative to adulthood. Two recent studies suggest that certain affective stimuli exert a greater influence on the risk-taking behavior of adolescents than adults. In an effort to directly test a dual-systems account of age differences in risk-taking behavior, Figner and colleagues developed two versions of the same risk-taking task (the Columbia Card Task), one of which was similar 
to traditional deliberative tasks (i.e., minimizing affective arousal), and the other designed to trigger affective involvement (Figner, Mackinlay, Wilkening, \& Weber, 2009). Both tasks presented participants with the opportunity to turn over cards revealing variable monetary gains and losses, after being explicitly informed about the magnitude and probability of the outcomes occurring. Thus, the tasks were equivalent in terms of the expected value of choice behavior. However, in contrast to the deliberative ("cold") version of the task, which required participants to choose the number of cards to turn over at the beginning of each trial, the affective ("hot") version instructed participants to turn over one card at a time, and presented feedback on gains and losses with each card. Thus, the cold version encouraged participants to rationally determine the optimal choice, whereas the hot version provided affective feedback (e.g., rewards and punishments) to guide the decision-making process. Consistent with traditional decision-making research, adolescents (ages 14-19) and adults (ages 20+) showed no differences in risktaking on the cold version of the task. In contrast, adolescents took significantly more risks than adults on the hot version of the task. Interestingly, although the effect was larger for adolescents than adults, both groups took more risks on the hot than the cold version of the task, consistent with the research reviewed earlier demonstrating the influence of affect on cognition in adult populations. Furthermore, across the age groups, risk-taking in the hot task was positively correlated with self-reported need for arousal and negatively correlated with information use, providing support for the assertion that the hot task indeed captured affective influences on decision-making. 
A more direct demonstration of the privileged role of socio-emotional stimuli, in particular, as an input to adolescent decision-making comes from an experimental study of peer context effects on risk-taking behavior (Gardner \& Steinberg, 2005). In this study, adolescents (mean age $=14$ ), youths (mean age $=$ 19), and adults (mean age $=37$ ) were tested on a computer driving task that mimicked the real-life experience of approaching a yellow light and deciding whether to stop and wait for the light to turn green again, or drive through the intersection and risk being hit by an unseen car. Peer context was manipulated by randomly assigning each group of three participants to play the game either individually (alone in the room), or with two same-aged peers in the room. When tested alone, the three age groups engaged in a comparable amount of risk-taking. However, when tested with peers in the room, adolescents and youth showed a significant increase in risk-taking, whereas adults did not. Specifically, adolescents scored twice as high on an index of risky driving when tested with their peers in the room, relative to when they were alone, whereas the college-aged group was approximately $50 \%$ riskier, and adults showed no differences in risky driving related to context. This experimental demonstration of heightened peer influence on risktaking in adolescence represents an important advance over prior studies correlating adolescents' risk behavior with behavior reported by their peers, findings that are subject to alternative explanations like selection or opportunity effects. At least in this one study, the presence of peers appeared to motivationally bias adolescents toward riskier behavior in a manner which was not apparent for adults. In the remainder of this chapter, we present a psychobiological model of adolescent 
development that offers a plausible account for this maturational window of increased susceptibility to peer influence on risk-taking.

\section{A Social Neuroscience Perspective on Adolescent Risk-Taking}

Developmental theories of risk-taking must account for two distinct trajectories observed in real-world behavior. First, risk-taking increases sharply from childhood to adolescence. Second, risk-taking steadily declines from late adolescence through the early adult years. Building on the dual-systems approaches described above, and incorporating recent evidence from developmental neuroscience, we argue that these two trajectories are related to normative maturational processes occurring in the brain over the course of adolescence and early adulthood. Specifically, we propose that risk-taking increases around the time of puberty due to changes in what we refer to as the brain's socio-emotional reward system, resulting in increased sensitivity to social and emotional stimuli and heightened motivation toward reward-seeking. Furthermore, we propose that risktaking decreases in the transition to adulthood due to gradual maturation of the brain's cognitive control system, which supports advancements in self-regulatory control over goal-directed behavior, as well as a decrease in reward sensitivity as individuals mature into adulthood. Because of the gap in maturational timing of the two systems, mid-adolescence represents a window of vulnerability to social and emotional influences toward risk-taking behavior that are relatively unchecked by an immature capacity for self-regulation. We now briefly review the neurobiological and behavioral evidence for this model; the reader is referred to 
the original presentation of the theory for a more extensive review (Steinberg, 2008).

\section{Development of the Socio-Emotional Reward System}

The emerging field of developmental neuroscience is quickly amassing evidence indicating dramatic structural and functional changes in the human brain occurring around the time of puberty. One of the most important of these developments is the remodeling of the dopaminergic system within limbic and paralimbic areas (including the amygdala, ventral striatum, orbitofrontal cortex, medial prefrontal cortex, and superior temporal sulcus), a network of interconnected regions that we refer to as the socio-emotional reward system. This network is centrally involved in the processing of social and emotional stimuli (e.g., face recognition, social judgments, social reasoning (Adolphs, 2003) and, importantly, includes neural circuits that mediate reward processing (Spear, 2009). Moreover, there is considerable overlap within this network between regions showing activation in response to social stimuli and regions that are differentially activated in response to variations in reward magnitude (e.g., the ventral striatum and medial frontal areas (Steinberg, 2008)).

Research with animal models points to a pattern of proliferation and pruning of dopamine receptors in the striatum and prefrontal cortex (PFC) during adolescence, a pattern which is more pronounced in males that females (Sisk \& Foster, 2004). Developmental changes in the mesocorticolimbic dopamine system, in particular, appear to parallel adolescent shifts in reward-related behavior (Spear, 2009). Briefly, this system includes dopamine neurons projecting from the mid- 
brain (substantia nigra (SN) and ventral tegmental area (VTA)) to the striatum (including the nucleus accumbens (NAcc)) and PFC. Converging evidence points to adolescent changes in dopamine receptor density and subsequent neurotransmission in the striatum and PFC. Dopamine receptor binding in the rat striatum peaks in adolescence at levels that are 30-45\% greater than levels observed in adulthood (e.g., Teicher et al., 1995). Furthermore, excitatory dopamine input to the PFC shows adolescent peaks in both rodent (Spear, 2009) and non-human primate populations (Rosenberg \& Lewis, 1995). Despite evidence for lower basal levels of dopamine release in adolescent (relative to adult) rats, adolescent rats evince greater dopamine release than adults in response to certain reward stimuli (Laviola, Pascucci, \& Pieretti, 2001).

Although the nature and implications of dopaminergic remodeling remain hotly contested, one account suggests that changes in the mesocorticolimbic dopamine system facilitate heightened sensitivity to rewards in adolescence, relative to childhood or adulthood (for discussion of alternative views, see Spear, 2009). Supporting this account, recent functional neuroimaging studies of age differences in reward processing have shown increased activation in adolescents of rewardrelevant subcortical regions (especially the nucleus accumbens) in response to reward receipt (e.g., Galvan et al., 2006). Note, however, that opposite results were found in an fMRI study of age differences in reward anticipation (rather than receipt), with adolescents showing decreased accumbens activation relative to adults (Bjork et al., 2004). Importantly, Galvan and colleagues (2006) also reported a significant correlation between accumbens activity and self-reported risk-taking 
behavior, providing convergent evidence that adolescent reward sensitivity contributes to a heightened propensity toward risky behavior. Furthermore, these neuroimaging findings are consistent with the observations from animal models of adolescence indicating elevated dopamine neurotransmission in frontostriatal circuits, described above (e.g., Laviola et al., 2001).

Consistent with this neuroimaging evidence for heightened reward sensitivity following puberty, adolescents report higher levels of sensation-seeking than children or adults, a pattern that appears more closely related to pubertal development than age (Martin et al., 2002). Moreover, this peak in sensation-seeking is mirrored in adolescent rodents, who show a marked increase in novelty-seeking behavior (Spear, 2009). Further evidence for curvilinear developmental changes in reward behavior comes from a recent study that examined age differences in reward processing, risk taking, and psychosocial maturity in a large population of individuals $(\mathrm{N}=935)$ ranging from 10 to 30 years old (for a complete review of study findings, see Steinberg, Cauffman, et al., in press). This study provided evidence for peaks (followed by declines) in early-to-middle adolescence of selfreported risk preference (Steinberg, Cauffman, et al., in press) and sensationseeking (Steinberg, Albert, et al., 2008), as well as behavioral indicators of reward sensitivity (on a modified version of the Iowa Gambling Task; Cauffman et al., in press) and preference for immediate over delayed rewards (Steinberg, Graham, et al., 2009). In contrast to this curvilinear pattern of development observed for measures of reward processing, age differences on measures of psychosocial maturity not directly related to reward processing (e.g., future orientation, impulse 
control, strategic planning) evinced a pattern of linear maturation extending across adolescence and into early adulthood (Steinberg, Cauffman, et al., in perss). We return to the latter findings in our discussion of the development of cognitive control.

In sum, evidence is beginning to accumulate suggesting that ongoing maturation of dopaminergic systems in adolescence contributes to changes in reward-system functioning coincident with heightened reward sensitivity and sensation-seeking. It should be noted, however, that this dopaminergic remodeling has not been directly linked to puberty-related gonadal hormones. Research with gonadectomized rodents demonstrates normative patterns of dopaminergic proliferation and pruning, indicating that such neural development is not steroiddependent (Spear, 2009). Puberty-coincident changes in dopaminergic systems may result from steroid-dependent processes (some of which are activated pre- or peri-natally), steroid-independent processes, or interactions between these processes (Steinberg, 2008).

Whereas there is little evidence for direct effects of gonadal hormones on dopaminergic remodeling, puberty-related increases in gonadal hormones have been linked to a proliferation of receptors for oxytocin within the limbic system, including such structures as the amygdala and nucleus accumbens (Spear, 2009). Oxytocin neurotransmission has been implicated in a variety of social behaviors, including facilitation of social bonding and recognition and memory for positive social stimuli (Insel \& Fernald, 2004). This evidence for puberty-related increases in gonadal hormones and oxytocin receptors is consistent with changes in a con- 
stellation of social behaviors observed in adolescence. In addition to reporting a spike in interest in opposite-sex relationships, adolescents begin to spend more time interacting with peers, and report the highest degree of happiness when they are doing so (e.g., Csikszentmihalyi, Larson, \& Prescott, 1977). This behavioral shift toward peer affiliation appears highly conserved across species; adolescent rats also spend more time than younger or older rats interacting with peers, while showing evidence that such interactions are highly rewarding (Spear, 2009). Moreover, recent developmental neuroimaging studies indicate that, relative to children and adults, adolescents show heightened activation within the socioemotional reward system in response to a variety of social stimuli, such as facial expressions and social feedback (Blakemore, 2008). Finally, consistent with adolescent reports of heightened emotional intensity, several recent studies have demonstrated puberty-related increases in emotional reactivity, as indexed by heightened startle reflex, pupillary reactivity, and cortisol and cardiovascular response (for a review, see Dahl \& Gunnar, 2009).

Taken together, this evidence for puberty-coincident remodeling of the brain's socio-emotional reward system and associated elevations in sensationseeking, reward salience, and sensitivity to social and emotional stimuli suggests a number of compelling possible answers to the question of why risk-taking behavior increases between childhood and adolescence. Based on the observed changes in adolescent reward system functioning, a first answer is simply that adolescents who have undergone remodeling of the dopaminergic system may be more responsive to the reward-value of risky choices than their younger counterparts. 
Building on this foundation, we propose a second mechanism: Not only are adolescents potentially more responsive to rewards, but due to puberty-related increases in sensitivity to social and emotional stimuli, this inclination toward approaching risky rewards is exacerbated when adolescents are in the presence of their peers. Such an explanation is consistent with the experimental evidence reviewed above showing that adolescents took twice as many risks on a driving simulation task when seated in-between two peers, compared to when they completed the task alone (Gardner \& Steinberg, 2005). Furthermore, empirical and theoretical work detailing the influence of affective states on decision-making suggests plausible neurobiological mechanisms for how such a peer effect might be instantiated in the brain. Recall our earlier description of a study demonstrating increased consummatory behavior in response to subliminal presentation of positively valenced, emotionally expressive faces (Winkielman et al., 2005). Pointing to the extensive structural overlap of neurobiological systems mediating processing of socio-emotional and incentive stimuli, the authors argued that positive emotional responses may sensitize incentive circuitry toward activation of approach responses to appetitive stimuli. Given evidence of puberty-related intensification of socio-emotional reactivity in adolescence, we suggest that adolescents are more likely than their younger counterparts to strongly activate such circuitry when in the presence of their peers, resulting in greater sensitization to the reward value of risky choices. At the conclusion of this chapter, we describe preliminary results of an ongoing neuroimaging study that begins to test these hypotheses. 
First, we turn to the question of why risk-taking declines as adolescents mature into adulthood.

\section{Development of the Cognitive Control System}

In contrast to the relatively sudden changes in social, emotional, and reward processing that occur around the time of puberty, cognitive capacities supporting mature self-regulation appear to develop in a gradual, linear pattern over the course of adolescence (and often into early adulthood) (Steinberg, 2008). A growing body of evidence from cognitive neuroscience suggests that these improvements in cognitive control are supported by structural and functional maturation of a phylogenetically recent brain system that includes the lateral PFC, parietal association cortices, and parts of the anterior cingulate cortex, as well as enhanced connectivity between this system and limbic circuitry (for a review, see Casey, Getz, \& Galvan, 2008).

Recent advances in structural neuroimaging techniques have permitted identification of two broad patterns of brain development occurring over the course of adolescence. First, after peaking between ages 10 and 12, the volume of gray matter in the frontal and parietal lobes decreases in the teenage years, a pattern commonly interpreted as reflective of synaptic pruning, the process by which infrequently activated neuronal connections are eliminated (Giedd, 2008). Importantly, among the last regions to complete the process of gray matter loss is the dorsolateral PFC (DLPFC), a region implicated as crucial for cognitive control by functional neuroimaging studies employing a variety of complex control tasks (Casey et al., 2008). A second feature of adolescent brain development is a 
whole-brain, linear increase in white matter that extends well into the twenties (Giedd, 2008). Increases in white matter volume are thought to reflect myelination, the process by which axons are wrapped in an insulating sheath, thereby supporting greater integrity and speed of neuronal transmission. Studies employing Diffusion Tensor Imaging (DTI), a technique for imaging white matter tracts and estimating their relative structural integrity, have provided further evidence for continued myelination over the course of adolescence (Giedd, 2008). Evidence of a prolonged course of myelination of neuronal connections within cortical regions, and between cortical and limbic regions, has led to predictions of improved processing efficiency on complex cognitive control tasks, as well as advances in the coordination of cognition and affect supporting goal-directed behavior. Indeed, such behavioral developments closely parallel the timetable for biological maturation we have just described.

Consistent with evidence for early adolescent synaptic pruning in the PFC, improvements in basic information processing and logical reasoning capacities thought to rely upon the PFC are still evident over the course of early-tomiddle adolescence, but show few maturational advancements beyond approximately age 16 (Steinberg, 2008). For instance, in the large-scale study of age differences in capacities contributing to risk-taking that we described previously, no developmental improvements in relatively simple measures of working memory or verbal fluency were evident after age 16 (Steinberg, Cauffman, et al., in press). When developmental gains in basic cognition do extend into later adolescence, they are typically seen on more demanding tasks with strong processing efficiency 
requirements, which likely rely on enhanced inter-cortical connectivity (Steinberg, 2008).

In contrast to the relatively limited adolescent gains in basic cognition, developmental improvements in higher-order executive functions known to simultaneously recruit multiple sub-regions of the PFC are evident across the course of adolescence and into early adulthood, consistent with the enhanced neural connectivity provided by ongoing myelination. For instance, improved performance is evident in late adolescence on tasks assessing response inhibition (e.g., Luna et al., 2001), strategic problem solving (e.g., Luciana, Collins, Olson, \& Schissel, 2009), and flexible rule use (e.g., Crone, Donohue, Honomichl, Wendelken, \& Bunge, 2006). Furthermore, consistent with gains in cortical-subcortical connectivity, improved coordination of cognitive and affective processes is also evident in late adolescence and early adulthood (Steinberg, 2008). For instance, self-report and behavioral evidence indicates a pattern of linear growth in impulse control extending through adolescence and into the twenties (Steinberg, Albert, et al., 2008).

The proposed link between structural brain maturation and gains in selfregulatory behavior is further supported by convergent evidence from functional neuroimaging studies of developmental differences in the neural correlates of cognitive control. Imaging studies utilizing a variety of cognitive control paradigms (e.g., Go-No/Go, Stroop, flanker tasks, antisaccade) suggest that adolescents recruit the control network - especially the DLPFC - less efficiently than adults (Casey et al., 2008). In general, adolescents show stronger activation than children of the DLPFC while engaging in cognitive control tasks, consistent with 
structural maturation of the region in early adolescence (e.g., Luna et al., 2001). In contrast, between adolescence and adulthood, differences in activation appear to reflect a process of refinement in the recruitment and coordination of structurally mature regions, rather than gross differences in level of activation. Specifically, adolescents show increasingly focal engagement of task-relevant regions supporting cognitive control, a functional advancement that may reflect the increased integrity and efficiency of inter-regional connections resulting from ongoing myelination (Durston et al., 2006).

\section{Interactions Between Reward and Control Systems}

To summarize thus far, we have presented evidence that dopaminergic remodeling coincident with puberty is associated with heightened sensitivity in the socio-emotional reward system in early adolescence, whereas synaptic pruning and myelination over the course of adolescence likely support gradual improvements in the efficiency of the cognitive control system. Is adolescent risk-taking then simply a matter of an overactive socio-emotional reward system dominating an immature control system? This idea of competitive dominance has been proposed as a mechanism contributing to risky or impulsive decision-making in contexts as diverse as drug use, cost/reward valuations, social information processing, and adolescent risk-taking (Steinberg, 2008). For instance, recent fMRI studies have shown correlations between activity in the socio-emotional reward system and preference for immediate over delayed rewards (McClure et al., 2004) and risky decision-making (Ernst et al., 2004). Furthermore, evidence from an experimental study utilizing transcranial magnetic stimulation demonstrated increased 
risk-taking following disruption of activity in the right DLPFC, a region consistently implicated in studies of cognitive control (Knoch et al., 2006). Together, these studies suggest that risk-taking may result from overactivity in the socioemotional reward system, underactivity in the cognitive control system, or a competitive imbalance between the two systems.

A second, complementary approach to understanding developmental changes in the interaction between the socio-emotional reward system and the cognitive control system is to focus on age differences in coordination between the two systems. That is, mature decision-making does not necessarily reflect developmental changes in the relative dominance of one system or the other, but rather the extent to which the two systems are simultaneously recruited and engage in "cross-talk" to produce a response that effectively integrates bottom-up (i.e., socio-emotional) and top-down (i.e., cognitive control) inputs. Given the steady gains in connectivity between cortical and subcortical regions observed over the course of adolescence, we would expect parallel gains in the capacity to integrate emotion and cognition. Indeed, adolescents evince gradual improvement of capacities reflecting self-regulatory control of emotionally-driven behavior, including impulse control, planning, and future orientation (Steinberg, 2008). However, improved coordination of emotion and cognition is not only reflected in the capacity to override emotional inputs, but also in the ability to adaptively utilize affective information to guide decision-making. For instance, research using a variety of affective learning paradigms (i.e., variants of the Iowa Gambling Task) demonstrates steady gains over the course of adolescence in the extent to which emo- 
tional feedback guides mature decision-making (e.g., Cauffman et al., in press). Most importantly for the present argument, this improved coordination of bottomup and top-down processing should facilitate growth in the capacity to regulate the influence of peers in risk-taking situations. Consistent with this prediction, a recent study demonstrated gradual, linear improvement in resistance to peer influence through at least age 18 (Steinberg \& Monahan, 2007).

Recent neuroimaging work also supports the proposed association between improved neural connectivity and parallel gains in resistance to peer influence. For instance, one innovative fMRI study assessed a group of 10-year-olds with varying degrees of self-reported resistance to peer influence on their responses to emotionally arousing video clips (i.e., angry vs. neutral biological motion) (Grosbras et al., 2007). Children reporting a relatively high degree of resistance to peer influence demonstrated greater functional connectivity in their responses to the emotional scenes, such that activity in motor-perception areas (i.e., dorsal premotor cortex) was correlated with activity in cognitive control regions (i.e., DLPFC). A second study demonstrated that individual differences within a group of 12-18 year-olds (after controlling for age) in resistance to peer influence were correlated with the extent of structural connectivity between prefrontal and premotor areas (Paus et al., in press). Although research relating age differences in structural and functional connectivity to maturation of behavior is in its infancy, these findings are nonetheless suggestive of the need to move beyond examining age differences in recruitment of specific regions and toward a consideration of developmental changes in coordinated neural activity. 


\section{Future Directions}

We are currently engaged in a program of research in our lab designed to test the neurobiological account of adolescent risk-taking proposed in this chapter. Building on experimental work showing age differences in the degree to which peer presence facilitates risky behavior (Gardner \& Steinberg, 2005), we have attempted to bring peer context into the scanner (Chein et al., 2009). Using an event-related fMRI design, we are examining age differences in neural activation at the moment of decision-making in two different risk-taking tasks (i.e., the Stoplight Game (Steinberg, Albert et al., 2008) and the Balloon Analogue Risk Task (Lejeuz et al., 2002)). To manipulate peer context, we measure task-related neural activation for each participant during two separate sessions. In one session, the participant completes the tasks while their peers are observing their performance from the scanner control room; in the other session, the participant completes the task with no observation. In each case, the participant is made aware of the condition. Consistent with our predictions, preliminary data indicates that adolescents activate socio-emotional reward regions (e.g., medial PFC, ventral striatum) more strongly when making risky decisions while being observed by their peers than when they do so alone. In contrast, these early data suggest that adults show few differences related to peer context in activation of socio-emotional reward centers, but instead show stronger activation of cognitive control regions (e.g., lateral PFC, posterior parietal) in the peer observation condition. Future analyses will also explore age differences in functional connectivity between cognitive control and so- 
cio-emotional reward regions that may mediate age differences in risk-taking behavior on these tasks.

\section{Conclusion}

Research efforts to account for developmental trajectories of risk-taking in adolescence have arrived at an exciting new stage. Moving beyond laboratory studies of age differences in "cool" cognitive processes related to risk perception and reasoning, new approaches have begun to incorporate insights from a rich literature describing the many pathways by which social and emotional factors influence the decision-making process. Combining these insights with emerging evidence from developmental neuroscience, we have outlined a theory that highlights a neuro-maturational gap between early adolescent remodeling of the socio-emotional reward system and a gradual, prolonged strengthening of the cognitive control system. Within this framework, middle adolescence represents a window of heightened vulnerability to peer influences toward risk-taking behavior. At a time when adolescents spend an increasing amount of time with their peers, research suggests that peer-related stimuli may sensitize the reward system to respond to the reward value of risky behavior. As the cognitive control system gradually matures over the course of the teenage years, adolescents grow in their capacity to coordinate affect and cognition, and to exercise self-regulation even in emotionally arousing situations. These capacities are reflected in gradual growth in the capacity to resist peer influence. 


\section{References}

Adolphs, R. (2003). Cognitive neuroscience of human social behavior. Nature Reviews Neuroscience, 4, 165-178.

Andersen, S., Thompson, A., Krenzel, E., \& Teicher, M. (2002). Pubertal changes in gonadal hormones do not underlie adolescent dopamine receptor overproduction. Psychoneuroendocrinology, 27, 683-691.

Azjen, L., \& Fishbein, M. (1980). Understanding attitudes and predicting behavior. Englewod Cliffs, NJ: Prentice Hall.

Becker, M.H. (1990). Theoretical models of adherence and strategies for improving adherence. In S.A. Shumaker, E.B. Schron, J.K. Ockene, C.T. Parker, J.L. Probstfield, \& J.M. Wolle (Eds.), Handbook of behavior change. New York: Springer.

Bjork, J.M., Knutson, B., Fong, G.W., Caggiano, D.M., Bennett, S.M., \& Hommer, D.W. (2004). Incentive-elicited brain activation in adolescents: Similarities and differences from young adults. Journal of Neuroscience, 24, 1793-1802.

Blakemore, S. (2008). The social brain in adolescence. Nature Reviews Neuroscience, 9, 267-277.

Brown, B. (2004). Adolescents' relationships with peers. In R. Lerner \& L. Steinberg (Eds.), Handbook of adolescent psychology (2 ${ }^{\text {nd }}$ ed.). New York: Wiley.

Casey, B.J., Getz, S., \& Galvan, A. (2008). The adolescent brain. Developmental Review, 28, 62-77. 
Cauffman, E., Shulman, E., Steinberg, L., Claus, E., Banich, M., Graham, S., \& Woolard, J. (in press). Age differences in affective decision making as indexed by performance on the Iowa Gambling Task. Developmental Psychology.

Chein, J., DiSorbo, A., Albert, D., O’Brien, L., Eagan, D.E., \& Steinberg, L. (2009, April). Neural markers of peer influence in adolescent risk taking. Paper presented at the biennial meeting of the Society for Research in Child Development, Denver, CO.

Crone, E. A., Wendelken, C., Donohue, S. E., Van Leijenhorst, L., \& Bunge, S. A. (2006). Neurocognitive development of the ability to manipulate information in working memory. Proceedings of the National Academy of Sciences, 103, 9325-9330

Csikszentmihalyi, M, Larson, R., \& Prescott, S. (1977). The ecology of adolescent activity and experience. Journal of Youth and Adolescence, 6, 281294.

Chassin, L., Hussong, A., \& Beltran, I. (2009). Adolescent substance use. In R. Lerner \& L. Steinberg (Eds.), Handbook of adolescent psychology, Vol.1. (3rd ed.) (pp. 723-763). Hoboken, NJ, US: John Wiley \& Sons Inc.

Dahl, R.E., \& Gunnar, M.R. (2009). Heightened stress responsiveness and emotional reactivity during pubertal maturation: Implications for psychopathology. Development and Psychopathology, 21, 1-6.

Damasio, A.R. (1994). Descartes' error: Emotion, reason, and the human brain. New York: Avon Books. 
Durston, S., Davidson, M., Tottenham, N., Galvan, A., Spicer, J., Fossella, J., et al. (2006). A shift from diffuse to focal activity with development. Developmental Science, 9, 1-20.

Ernst, M., Nelson, E., Jazbec, S., McClure, E., Monk, C., Blair, R., et al. (2004). Choice selection and reward anticipation: An fMRI study. Neuropsychologia, 42, 1585-1597.

Fischhoff, B. (2008). Assessing adolescent decision-making competence. Developmental Review, 28, 12-28.

Figner, B., Mackinlay, R.J., Wilkening, F., \& Weber, E.U. (2009). Affective and deliberative processes in risky choice: Age differences in risk taking in the Columbia Card Task. Journal of Experimental Psychology: Learning, Memory, and Cognition, 35, 709-730.

Galvan, A., Hare, T., Parra, C., Penn, J., Voss, H., Glover, G., \& Casey, B.J. (2006). Earlier development of the accumbens relative to the orbitofrontal cortex might underlie risk-taking behavior in adolescents. Journal of Neuroscience, 26, 6885-6892.

Gardner. M., \& Steinberg, L. (2005). Peer influence on risk taking, risk preference, and risky decision making in adolescence and adulthood: An experimental study. Developmental Psychology, 41, 625-635.

Giedd, J.N. (2008). The teen brain: Insights from neuroimaging. Journal of Adolescent Health, 42, 335-343. 
Grosbras, M., Jansen, M., Leonard, G., McIntosh, A., Osswald, K., Poulsen, C., et al. (2007). Neural mechanisms of resistance to peer influence in early adolescence. Journal of Neuroscience, 27, 8040-8045.

Insel, T., \& Fernald, R. (2004). How the brain processes social information: Searching for the social brain. Annual Review of Neuroscience, 27, 697722.

Jaccard, J., Blanton, H., \& Dodge, T. (2005). Peer influences on risk behavior: An analysis of the effects of a close friend. Developmental Psychology, 41, $135-147$.

Jessor, R., \& Jessor, S.L. (1977). Problem behavior and psychosocial development: A longitudinal study of youth. New York: Academic Press.

Johnson, E.J., \& Tversky, A. (1983). Affect, generalization, and the perception of risk. Journal of Personality and Social Psychology, 45, 20-31.

Knoch, D., Gianotti, L., Pascual-Leone, A., Treyer, V., Regard, M., Hohmann, M., et al. (2006). Disruption of right prefrontal cortex by low-frequency transcranial magnetic stimulation induces risk-taking behavior. Journal of Neuroscience, 26, 6469-6472.

Laviola, G., Pascucci, T., Pieretti, S. (2001). Striatal dopamine sensitization to Damphetamine in periadolescent but not in adult rats. Pharmacology, Biochemistry and Behavior, 68,115-124.

Levin, I.P., Hart, S.S., \& Weller, J.A. (2007). Stability of choice in a risky decision-making task: A 3-year longitudinal study with children and adults. Journal of Behavioral Decision Making, 23, 241-252. 
Loewenstein, G.F., Weber, E.U., Hsee, C.K., \& Welch, N. (2001). Risk as feelings. Psychological Bulletin, 127, 267-286.

Luciana, M., Collins, P.F., Olson, E.A., \& Schissel, A.M. (2009). Tower of London performance in healthy adolescents: The development of planning skills and associations with self-reported inattention and impulsivity. Developmental Neuropsychology, 34, 1-15.

Luna, B., Thulborn, K., Munoz, d., Merriam, E., Garver, K., Minshew, N., et al. (2001). Maturation of widely distributed brain function subserves cognitive development. Neuroimage, 13, 786-793.

Martin, C.A., Kelly, T.H., Rayens, M., Brogli, B.R., Brenzel, A., Smith, W.J., et al. (2002). Sensation seeking, puberty and nicotine, alcohol and marijuana use in adolescence. Journal of the American Academy of Child and Adolescent Psychiatry, 41, 1495-1502.

McClure, S., Laibson, D., Loewenstein, g., \& Cohen, J. (2004). Separate neural systems value immediate and delayed monetary rewards. Science, 306, 503-507.

Paus, T., Toro, R., Leonard, G., Lerner, J., Lerner, R., Perron, M., et al. (in press). Morphological properties of the action-observation network in adolescents with low and high resistance to peer influence. Social Neuroscience.

Reyna, V.F., \& Farley, F. (2006). Risk and rationality in adolescent decision making: Implications for theory, practice, and public policy. Psychological Science in the Public Interest, 7, 1-44. 
Rosenberg, D., Lewis, D.A. (1995). Postnatal maturation of the dopaminergic innervation of monkey prefrontal and motor cortices: A tyrosine hydroxylase immunohistochemical analysis. Journal of Comparative Neurology, $358,383-400$.

Schwarz, N., \& Clore, G.L. (1983). Mood, misattribution, and judgments of wellbeing: Informative and directive functions of affective states. Journal of Personality and Social Psychology, 45, 513-523.

Sisk, C., \& Foster, D. (2004). The neural basis of puberty and adolescence. Nature Neuroscience, 7, 1041-1047.

Simons-Morton, B., Lerner, N., \& Singer, J. (2005). The observed effects of teenage passengers on the risky driving behavior of teenage drivers. Accident Analysis and Prevention, 37, 973-982.

Spear, L. (2009). The behavioral neuroscience of adolescence. New York: Norton.

Steinberg, L. (2008) A social neuroscience perspective on adolescent risk-taking. Developmental Review, 28, 78-106.

Steinberg, L., \& Cauffman, E. (1996). Maturity of judgment in adolescence: Psychosocial factors in adolescent decision making. Law and Human Behavior, 20, 249-272.

Steinberg, L., Albert, D., Cauffman, E., Banich, M., Graham, S., \& Woolard, J. (2008). Age differences in sensation seeking and impulsivity as indexed by behavior and self-report: Evidence for a dual systems model. Developmental Psychology, 44, 1764-1777. 
Steinberg, L., Cauffman, E., Woolard, J., Graham, S., \& Banich, M. (in press). Are adolescents less mature than adults? Minors' access to abortion, the juvenile death penalty, and the alleged APA "flip-flop". American Psychologist.

Steinberg, L., Graham, S., O'Brien, L., Woolard, J., Cauffman, E., \& Banich, M. (2009). Age differences in future orientation and delay discounting. Child Development, $80,28-44$.

Steinberg, L., \& Monahan, K. (2007). Age differences in resistance to peer influence. Developmental Psychology, 43, 1531-1543.

Teicher, M., Andersen, S., Hostetter, J. (1995). Evidence for dopamine receptor pruning between adolescence and adulthood in striatum but not nucleus accumbens. Developmental Brain Research, 89, 167-172.

Winkielman, P., Berridge, K.C., \& Willbarger, J. (2005). Unconscious affective reactions to masked happy versus angry faces influence consumption behavior and judgments of value. Personality and Social Psychology Bulletin, 1, 121-135.

Winkielman, P., Knutson, B., Paulus, M., \& Trujillo, J.L. (2007). Affective influence on judgments and decisions: Moving toward core mechanisms. Review of General Psychology, 11, 179-192.

Zajonc, R.B. (1980). Feeling and thinking: Preferences need no inferences. American Psychologist, 35, 151-175.

Zimring, F.E. (1998). American youth violence. Oxford, England: Oxford University Press. 